Meta

Journal des traducteurs

Translators' Journal

\title{
La terminologie scientifique bilingue : étude comparative pilote
}

\section{Christine Bagge}

Volume 27, numéro 4, décembre 1982

URI : https://id.erudit.org/iderudit/001945ar

DOI : https://doi.org/10.7202/001945ar

Aller au sommaire du numéro

Éditeur(s)

Les Presses de l'Université de Montréal

ISSN

0026-0452 (imprimé)

1492-1421 (numérique)

Découvrir la revue

Citer cet article

Bagge, C. (1982). La terminologie scientifique bilingue : étude comparative pilote. Meta, 27(4), 431-438. https://doi.org/10.7202/001945ar d'utilisation que vous pouvez consulter en ligne.

https://apropos.erudit.org/fr/usagers/politique-dutilisation/ 
WILSS, W., (1979a): "Probleme und Perspektiven der Studiengänge Übersetzen und Dolmetschen and der Universität des Saarlandes", in Lebende Sprachen, 4/1979, p. 147-150.

WILSS, W., (1979b) : "Fachsprache und Übersetzen" in FelBer H., F. LANG and G. WERSIG (eds.), Gedenkschrift für Univ.-Prof. Dr. Eugen Wüster, München, p. 177-195.

WILSS, W., (1982): The Science of Translation. Problems and Methods, Tübingen.

WUSTER, E., (1931/1970) : Internationale Sprachnormung in der Technik, (3rd ed. 1970), Bonn.

WÜSTER, E., (1963): "Die Struktur der sprachlichen Begriffswelt und ihre Darstellung in Wörterbüchern," in Proceedings of the $3^{\text {rd }}$ Congress of FIT, Oxford, p. 415-443. See also WÜSTER, E., (1959): "Die Struktur der sprachlichen Begriffswelt und ihre Darstellung in Wörterbüchern" in Studium Generale 12, p. 615-627.

WÜSTER, E., (1968): The Machine Tool, London.

WÜSTER, E., (1969): "Die vier Dimensionen der Terminologiearbeit" in Mitteilungsblatt für Übersetzer und Dolmetscher, 2/1969, p. 1-6, 5/1969, p. 1-5.

WÜSTER, E., (1971) : Die Bedeutung des Internationalen Informationszentrums für Terminologie, unpublished ms., Wüster Library, Infoterm, W 574.

WÜSTER, E., (1972): "Die allgemeine Terminologielehre - Ein Grenzgebiet zwischen Sprachwissenschaft, Logik, Ontologie, Informatik und den Sachwissenschaften", in Proceedings, vol. III, $3^{\text {rd }}$ Congress of Applied Linguistics, Copenhagen, p. 640-655.

WUUSTER, E., (1974) : The Road to Infoterm. Infoterm Series 1, München.

WÜSTER, E., (1975): "Die Ausbildung in Terminologie und terminologischer Lexikographie" in Lebende Sprachen, 2/1975, p. 33-37.

WÜSTER, E., (1979): (Bauer, L., ed.), Einführung in die Allgemeine Terminologielehre und Terminologische Lexikographie, Wien-New York.

WÜSTER, E., (1980) : "Introduction (Fragment of a Draft)", in SAGER J.S. and J.A. FishMAN (eds.), International Journal of the Sociology of Language 23, The Hague, p. 15.

\section{LA TERMINOLOGIE SCIENTIFIQUE BILINGUE : ÉTUDE COMPARATIVE PILOTE}

[...] toutes les fois qu'il $\mathrm{y}$ a situation commune, ou semblable, il peut y avoir traduction. Ceci explique en particulier pourquoi, plus les traits sémantiquement pertinents d'une situation sont limitativement décrits, définis et comptés (ce qui est le cas dans tous les domaines scientifiques), plus la traduction est possible et complète, et ceci, quels que soient l'écart et même l'incommensurabilité des syntaxes entre langue-source et langue-cible.

D'après G. Mounin (1980: 265), donc, dans le domaine scientifique, plus que dans tout autre, il serait possible de créer une terminologie française qui ne doive rien à l'anglaise (et vice versa) puisque la réalité qu'elles décrivent est «commune». Georges Mounin n'est pas le seul à avoir insisté sur ce fait. T. H. Savory, entre autres, le remarque également et va même jusqu'à dire qu'il est souvent difficile, dans le contexte des sciences, de distinguer l'original de sa traduction. Il écrit: $\alpha[\ldots]$ it has been said that no one term in French corresponds in all circumstances to one and the same term in English, except of course in scientific [...] writing. " (Savory 1967:140).

Il est un fait que les sciences sont probablement le domaine où la distance entre le signifié et le référent, pour reprendre la terminologie saussurienne, est la plus courte. Puisque le référent est «limitativement décrit et défini», le signifié est très précis. Contrairement à ce qui se passe dans la langue courante, donc, en terminologie scientifique, le signifiant, dans la majorité des cas, définit le signifié dans sa totalité. Or, comme le montre la figure 1 , plus la distance référent-signifié-signifiant est courte, plus l'équivalence entre le signifiant fran- 
çais et le signifiant anglais est grande. Autrement dit, l'équivalence entre les deux terminologies est inversement proportionnelle à la distance qui sépare le signifiant du référent.

Il serait toutefois faux de considérer que les sciences sont totalement objectives. Une erreur courante consiste à confondre l'interprétation de la réalité par les scientifiques avec la réalité elle-même. Pour élaborer les postulats fondamentaux de la mécanique quantique, par exemple, le Danois Niels Bohr et l'Allemand Werner Heisenberg ont travaillé ensemble. Cependant, c'est le formalisme logique qui intéressait W. Heisenberg, alors que N. Bohr a préféré opter pour une optique plus pragmatique. En fin de compte; ils ont tous deux appliqué la théorie des quanta, mais de façon légèrement différente. Et pourtant ils décrivaient la même réalité ${ }^{1}$. Cet exemple montre bien que l'interprétation de la réalité par la science n'est pas totalement objective et universelle. Il est donc normal de s'attendre à ce que, bien qu'à un degré moindre que la langue courante, la terminologie scientifique découpe la réalité d'une façon qui varie d'une langue à l'autre. Goetschalckx, alors président du bureau de terminologie des commissions européennes, a écrit: «Au fil de nombreuses traductions, de centaines et centaines de pages, $\mathrm{j}$ 'ai buté sur des problèmes de traduction bien sûr, mais aussi sur des problèmes de langue et même de civilisation [...] des termes et des expressions reflètent $[. .$.$] le caractère propre de$ ceux qui les emploient.» (1973: 261). Le but de cette recherche sera donc d'étudier sur des exemples aussi précis et aussi probants que possible les caractéristiques qui différencient la terminologie scientifique française de l'anglaise, de déterminer quelles sont les attitudes mentales et culturelles qui les gouvernent et d'essayer d'en dégager une certaine marche à suivre qui pourrait être utile lors de la création de néologismes.

Figure 1

Variation du degré d'équivalence

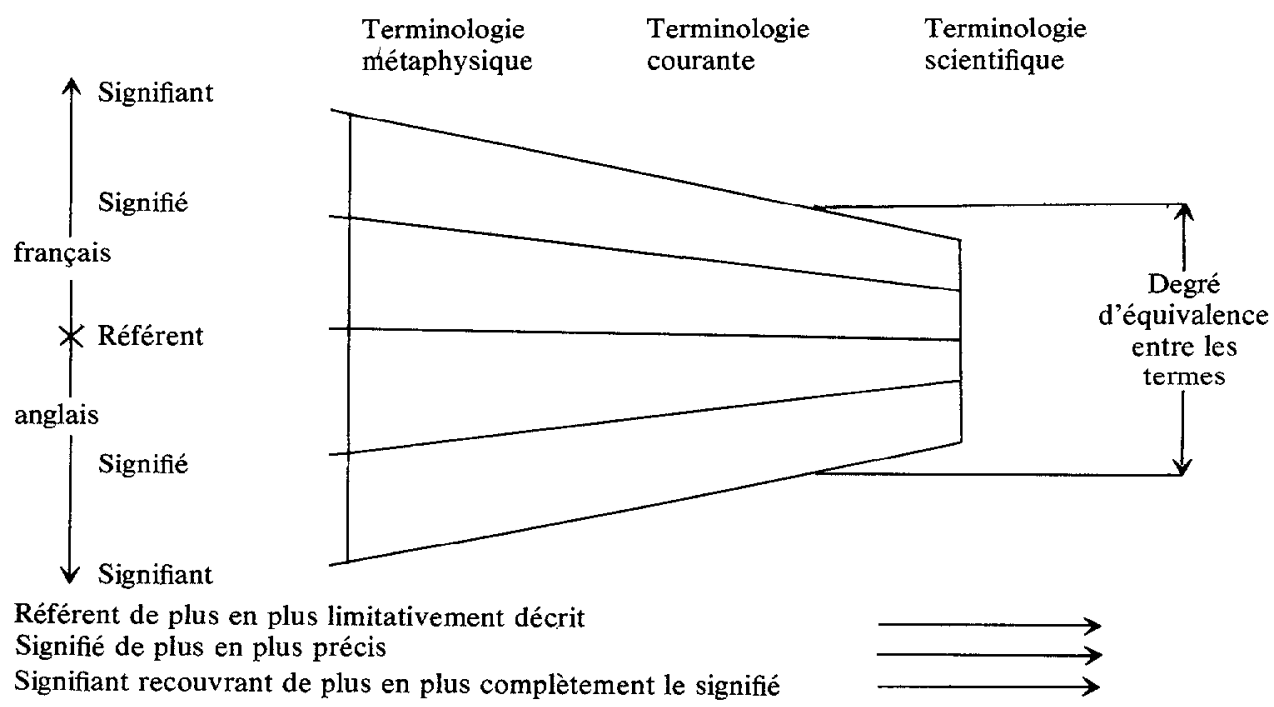

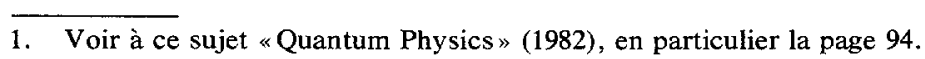


Cet article cependant n'est qu'une étude pilote et se concentrera sur un problème particulier relatif à cette recherche. Pour restreindre le domaine qui sera étudié, il est tout d'abord nécessaire de déterminer avec précision les possibilités de rapport qui existent entre les signifiés et les signifiants des deux langues. La figure 2 permettra d'effectuer cette démarche plus facilement. Il y a donc quatre possibilités représentées dans le tableau 1. Cet article traitera du groupe $\mathrm{A}$, c'est-à-dire des cas où une différence morphologique s'accompagne de divergences au niveau du découpage de la réalité.

Un corpus de 600 termes relevés au hasard dans différents domaines (la botanique, la géologie, la biologie, la physique, la chimie, l'astronomie, l'écologie) a été étudié. "Terme» dans le contexte de cet article est pris dans le sens d'unité sémantique nominale, c'est-à-dire substantif ou syntagme nominal. Étant donné que comme le notent T. H. Savory (1967: 26) et A. Phal (1970: 103), la différence qu'il y a entre la langue parlée et la langue écrite dans la vie quotidienne n'existe pas dans le domaine des sciences, la notion de niveau de langue n'intervient pas. Il y a, en revanche, des variations extrêmement importantes selon le degré de connaissance du locuteur et du récepteur. Bien que celui-ci affecte le texte dans son ensemble plutôt que la terminologie, mis à part le problème relativement rare de la synonymie, il est tout de même important de mentionner que ces 600 termes ont été relevés dans des articles de vulgarisation parus dans diverses revues spécialisées.

Il est également de rigueur de préciser qu'il s'agit là de termes scientifiques et non techniques. La distinction entre science et technique est pratiquement impossible à établir de façon absolue. Dans le cadre de cet article, la technique sera considérée comme l'application des sciences, mais il est bien évident que les échanges entre les sciences et la technique sont incessants et se font dans les deux sens. Sur le plan de la terminologie, la différence entre les deux peut se résumer de la façon suivante: tous les termes scientifiques font partie de la terminologie technique qui, elle, est plus vaste (figure 3). La paroi entre les deux n'est pas étanche (c'est ce que représentent les pointillés du cercle intérieur).

La première démarche a consisté à former deux groupes selon la morphologie des termes: $\mathrm{AD}$ et $\mathrm{BC}$ d'après le tableau 1. Ensuite le groupe $\mathrm{AD}$ a été scindé en $A$ et $D$. Finalement le groupe $A$ a été étudié de près pour essayer d'analyser les différences de découpage de la réalité.

Sur ces 600 termes, 411 présentaient des signifiants identiques, c'est-àdire des signifiants dont la morphologie ne différait que par les ajustements orthographiques indispensables à leur inclusion dans la langue en question. Ce haut pourcentage n'est en rien surprenant puisque l'histoire explique ce phénomène qui est reconnu depuis longtemps. D'une part la communication entre scientifiques de différents pays a toujours existé et a toujours été considérée comme essentielle. Les hommes de science ont essayé autant que possible de conjuguer leurs efforts de systématisation et ils forment en quelque sorte une communauté à part qui a son langage propre. D'autre part, comme l'indique D. Tchekoff (1971: 36) "le développement toujours plus rapide de la science [...] réclame la formation de mots nouveaux pour exprimer les idées nouvelles. 
A cet effet les langues disposent d'un lexique international formé d'emprunts au grec, qu'elles accommodent ensuite à leurs nécessités linguistiques particulières. Traditionnellement, c'est à partir de leur transcription en latin que sont formés les emprunts au grec dans les langues modernes à alphabet latin. " C'est en langues anciennes que se sont écrits les premiers traités scientifiques et c'est à ces langues que la terminologie a eu recours lorsqu'il s'est agi de trouver des termes vernaculaires. Ceci lui confère un haut degré de motivation ${ }^{2}$ et c'est

Figure 2

Rapports entre les signifiés et les signifiants des deux langues

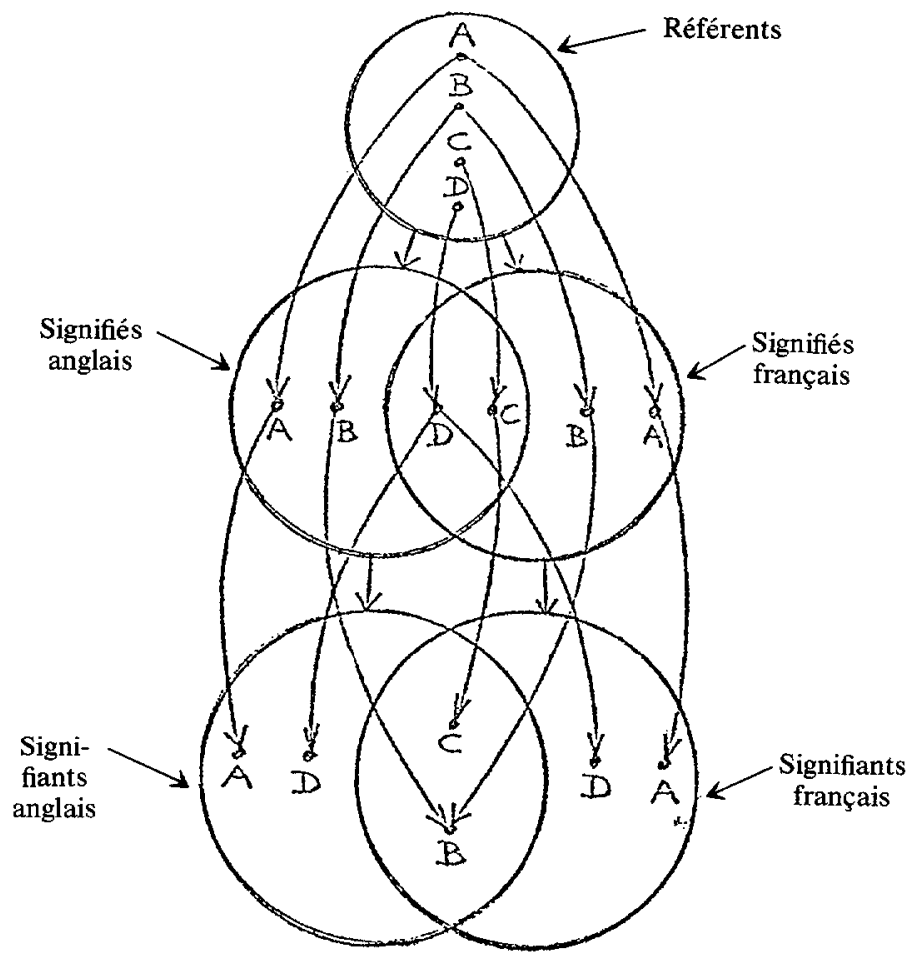

Tableau 1

Rapports possibles entre les signifiés et les signifiants des deux langues.

\begin{tabular}{cllll}
\hline & A & B & C & D \\
\hline Signifiés & Différents & Différents & Identiques & Identiques \\
\hline Signifiants & Différents & Identiques & Identiques & Différents \\
\hline
\end{tabular}

2. Le terme «motivation» est pris dans le sens que lui donne R. Dubuc (1978: 77) dans le contexte de la terminologie : "[...] la motivation s'attache à la valeur significative de l'étiquette elle-même. Un terme motivé est celui dont l'usager peut saisir le sens au moins sommairement par l'analyse de ses composants.» 


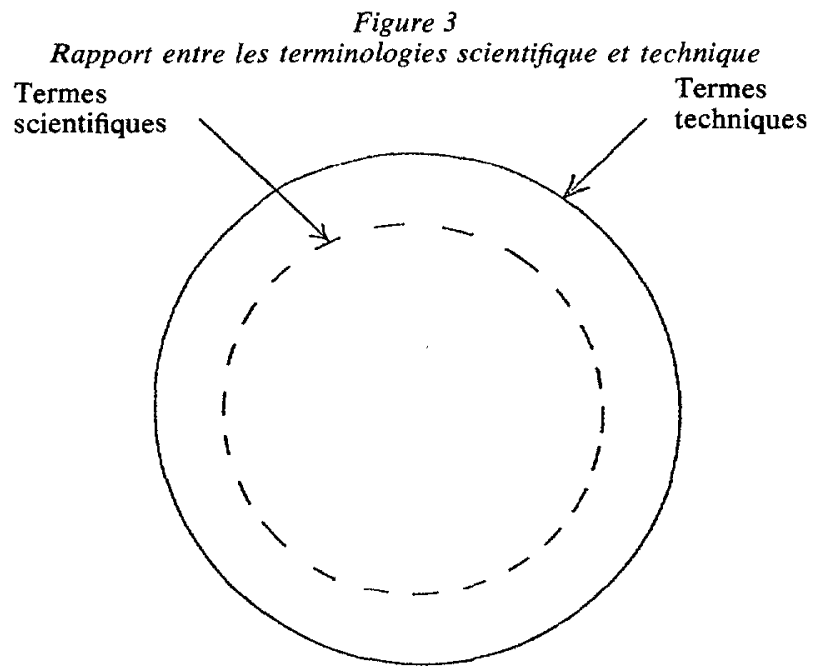

toujours de ce point de vue qu'elle a été étudiée. Pour interpréter la relation qui existe entre morphologie et sémantique (le sens des racines, des préfixes, des suffixes, etc...) on peut se rapporter par exemple à T. H. Savory (1967), à W. E. Flood (1960), à L. Hogben (1971) et surtout à H. Marchand (1969), à J. Cellard (1980) et à A. Cailleux et J. Komorn (1981).

Ceci laisse donc 189 termes pour former le groupe AD, soit environ $30 \%$ du nombre total de termes. Ce groupe est formé de 49 termes simples (composés d'un seul mot) et de 140 syntagmes. Comme il a déjà été indiqué, c'est avant tout du groupe A que traite cet article. Il est tout de même intéressant de mentionner au passage la façon dont s'est opérée la scission A-D, afin de comprendre avec plus de précision quels termes font partie du groupe $A$.

Dans le groupe D se classent 28 termes simples et 125 syntagmes. Ces termes simples sont des termes dont l'origine linguistique diffère pour des raisons historiques mais dont le sens original est le même, c'est-à-dire qui ne présentent pas de diversités au niveau du découpage de la réalité (blood/sang). Il s'agit également d'emprunts intérieurs dont le sens étymologique varie d'une langue à l'autre mais qui, au cours de leur séjour dans le langage courant ont fini par recouvrir la même notion (blanket/couverture, en physique nucléaire). En ce qui concerne les syntagmes du groupe $\mathrm{D}$, deux facteurs majeurs interviennent pour leur donner des signifiants différents. Tout d'abord, l'ordre séquentiel normal de la détermination est en anglais l'inverse de celui du français, mais cette inversion ne s'accompagne généralement pas d'une modification des signifiés (abdominal cavity/cavité abdominale). D'autre part, le fait qu'en anglais le nom peut s'employer comme adjectif mais que ce n'est pas le cas en français entraîne certaines différences sur le plan morphologique, notamment l'emploi d'adjectifs savants (food chain/chaîne trophique) ou de prépositions (action potential/potentiel d'action) en français. Premièrement, si l'adjectif savant français, comme dans l'exemple cité, a le même sens étymologique que le nom anglais utilisé comme adjectif, on peut considérer que le découpage de la réalité est le même dans les deux langues. Deuxièmement, le choix des prépositions est 
gouverné par le niveau sémantique, c'est-à-dire qu'à chaque préposition (de, en, à...) correspond une valeur sémantique particulière, conférée au terme par une phrase «sous-tendante». Cependant cette valeur sémantique ne varie généralement pas d'une langue à l'autre. Se classent dans le groupe $\mathrm{D}$, donc, les syntagmes dont la modification du signifiant n'est dictée que par les contraintes de la langue. Le groupe A, par contre, comprend les syntagmes dont les différences sur le plan morphologique, bien que toujours sujettes aux contraintes de la langue, s'accompagnent de variations au niveau du signifié.

Il reste ainsi dans le groupe A, 16 termes simples et 15 syntagmes, soit un total de 31 termes, environ $5 \%$ du nombre total de termes constituant l'échantillon étudié. Ces termes se classent à leur tour dans diverses catégories selon le genre de modulation lexicale ${ }^{3}$ auquel ils appartiennent, selon la façon dont ils diffèrent dans leur découpage de la réalité.

\section{I - L'abstrait et le concret}

Ce qui distingue certains de ces termes français des termes anglais correspondants, c'est qu'ils se situent à un niveau d'abstraction supérieur. C'està-dire que l'anglais exprime les notions de façon plus nette et précise que le français.

Exemple: stand/peuplement $($ En botanique $=$ ensemble des végétaux ou des arbres qui croissent en une même région, sur un même terrain)

Un terme simple et deux syntagmes se trouvent dans cette catégorie.

\section{II - Cause et effet}

Cette catégorie compte deux termes et trois syntagmes.

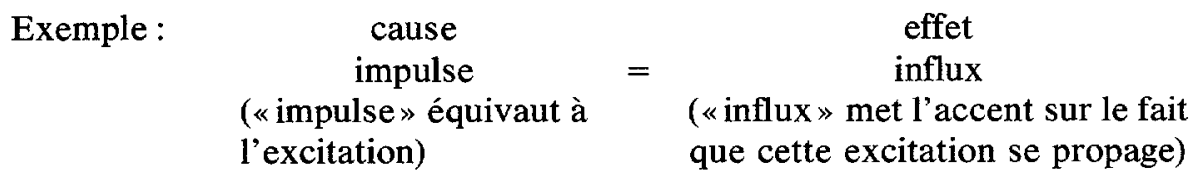

\section{III - Renversement du point de vue}

Il s'agit de cas où la vision de la réalité en anglais est l'inverse de la vision de la même réalité en français. Un exemple de ce genre de modulation se trouve dans :

$$
\text { terminal moraine/moraine frontale }
$$

Six syntagmes et un terme simple entrent dans cette catégorie.

\section{IV - Une caractéristique pour une autre}

Le signifiant recouvre parfois partiellement le signifié et dans ce cas-là, la caractéristique mise en évidence par le terme n'est pas toujours la même dans les deux langues:

$$
\text { Exemples: } \quad \text { digestive system = appareil digestif }
$$

(Le français insiste sur le fait que ces organes se complètent pour remplir leur fonction, alors que l'anglais ne fait qu'indiquer qu'ils sont apparentés.)

$$
\text { carbon dioxide }=\text { gaz carbonique }
$$

3. Le terme «modulation lexicale» est utilisé dans le sens que lui donnent Vinay et Darbelnet (1977: 88) : représentation «de la même réalité sous un jour différent ». 
(Pour l'anglais, il s'agit d'un oxyde contenant, par molécule, deux atomes d'oxygène et du carbone. Dans le cas du français, par contre, il est spécifié que c'est un gaz et si «carbonique» implique combinaison du carbone et de l'oxygène, le nombre d'atomes d'oxygène par molécule n'est pas précisé. Le terme "dioxyde de carbone» existe également mais n'est pas le terme généralement utilisé pour nommer ce gaz. Il est intéressant de noter au passage que ce genre de phénomène se produit même en chimie où la terminologie forme un des réseaux des plus structurés de la terminologie scientifique.)

Trois syntagmes et deux termes simples se trouvent dans cette catégorie.

$V$-Différentes images

Il s'agit d'une part de termes dont la différence de sens étymologique reflète une vision particulière de la réalité. C'est dans cette catégorie que se classe le plus grand nombre des termes simples.

Exemple :

$$
\begin{aligned}
& \text { computer }=\text { ordinateur } \\
& \text { (latin, computare }=\text { calculer) (latin, ordinare }= \\
& \text { mettre de l'ordre) }
\end{aligned}
$$

D'autre part, puisque la terminologie scientifique fait parfois appel au langage courant par le biais de la métaphore, il n'est pas surprenant qu'elle crée alors des termes qui représentent de façon variée la même réalité métalinguistique. Un exemple de ce genre de modulation a été trouvé :

planetary tides / vents planétaires (astronomie)

Tableau 2

Groupe A

\begin{tabular}{lccccc}
\hline & $\begin{array}{c}\text { I } \\
\text { Abstrait/ } \\
\text { concret }\end{array}$ & $\begin{array}{c}\text { II } \\
\text { Cause/effet }\end{array}$ & $\begin{array}{c}\text { III } \\
\text { Renversement } \\
\text { du point de vue }\end{array}$ & $\begin{array}{c}\text { Une caractéristique } \\
\text { pour une autre }\end{array}$ & $\begin{array}{c}\text { Différentes } \\
\text { images }\end{array}$ \\
\hline $\begin{array}{l}\text { Termes } \\
\text { simples }\end{array}$ & 1 & 2 & 1 & 2 & 10 \\
Syntagmes & 2 & 3 & 6 & 3 & 1 \\
Total & 3 & 5 & 7 & 5 & 11 \\
\hline
\end{tabular}

L'examen d'un échantillon plus important permettrait sans doute de délimiter un nombre plus grand de catégories. En ce qui concerne cette étude pilote, les résultats obtenus sont récapitulés dans le tableau 2.

La terminologie scientifique n'est pas aussi universelle qu' on l'a souvent laissé entendre. Cette étude pilote a montré que la modulation lexicale y joue un rôle non négligeable. Il serait intéressant de recenser de façon plus complète les différents genres de modulation qui interviennent en terminologie scientifique et de les comparer quantativement et qualitativement avec ceux qui existent dans le langage courant. Il serait sans doute également souhaitable de faire une étude comparative entre les sciences elles-mêmes, c'est-à-dire d'étudier séparément les terminologies de différents domaines et d'en comparer les résultats. I1 serait alors possible de déterminer quelles sciences se rapprochent ou s'éloignent 
de la langue courante au niveau des différences de découpage de la réalité entre les deux langues.

D'autre part, la présence de ces modulations prouve que malgré le haut degré de similarité qui existe entre les deux terminologies, il est dangereux, lors de la création de néologismes, de traduire littéralement. Il est impératif de retourner au référent, c'est-à-dire d'avoir directement connaissance de la réalité décrite sans passer forcément par tout ce que l'autre langue lui associe comme concepts.

Finalement, cette étude pilote n'a considéré qu'un aspect du problème et ne s'est basée que sur un corpus relativement restreint, mais son but principal est d'indiquer la direction que prendra une recherche plus poussée dans ce domaine.

\section{Christine BAGge}

\section{BIBLIOGRAPHIE}

CAILLEUX, André et Jean KOMORN (1981): Dictionnaire des racines scientifiques, Paris, C.D.U. et SEDES réunis.

CELLARD, J. (1980): Les 500 racines grecques et latines les plus importantes du vocabulaire français, Paris-Gembloux, Duculot.

DUBUC, Robert (1978) : Manuel pratique de terminologie, Montréal, Linguatech.

FLOOD, W.E. (1960): Scientific Words: their Structure and Meaning, London, Oldbourne.

GOETSCHALCKX, J. (1973): «Essai d'étude comparative des locutions et termes d'un glossaire technique plurilingue», Meta XVIII-1/2, p. 261-267.

HOGBEN, L., F.R.S. (1971): The Vocabulary of Science, London, Heinemann, $1^{\text {re }}$ édition 1969.

MARCHAND, H. (1969): The Categories and Types of Present-Day English Word-Formation, München, Beck.

MOUNIN, Georges (1980): les Problèmes théoriques de la traduction, Saint-Amand, Gallimard.

PHAL, A. (1970) : «Le vocabulaire général d'orientation scientifique : essai de définition et méthode, d'enquête", les Langues de spécialité, Strasbourg, AIDELA.

"Quantum Physics» (1982): The Economist, 3-9 April, p. 93-98.

SAVORY, T.H. (1967): The Language of Science, London, Deutsch, 1re édition 1953.

TCHEKOFF, C. (1971) : «Les formations savantes gréco-latines en français, anglais, italien, espagnol, allemand et russe», la Linguistique 7, p. 36-49.

VINAY, J.-P. et Jean DARBELNET (1977) : Stylistique comparée, Montréal, Beauchemin,

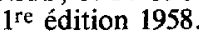

